\title{
FAITS ET CHIFFRES
}

\section{Les contrats de commercialisation des animaux en France. Une réponse à la variabilité du prix du porc?}

Animal marketing contracts in France - an answer to hog price variability?

Estelle Antoine, Marie-Alix Roussillon, Vincent Legendre et Michel Rieu

\section{(2) OpenEdition \\ Journals}

Édition électronique

URL : http://journals.openedition.org/economierurale/4564

DOI : $10.4000 /$ economierurale. 4564

ISSN : 2105-2581

\section{Éditeur}

Société Française d'Économie Rurale (SFER)

Édition imprimée

Date de publication : 15 février 2015

Pagination : 53-72

ISSN : 0013-0559

Référence électronique

Estelle Antoine, Marie-Alix Roussillon, Vincent Legendre et Michel Rieu, «Les contrats de commercialisation des animaux en France. Une réponse à la variabilité du prix du porc? ", Économie rurale [En ligne], 345 | Janvier-février 2015, mis en ligne le 15 février 2017, consulté le 10 décembre 2020. URL : http://journals.openedition.org/economierurale/4564 ; DOI : https://doi.org/10.4000/ economierurale. 4564 


\title{
Les contrats de commercialisation des animaux en France Une réponse à la variabilité du prix du porc ?
}

\author{
Estelle ANTOINE • Marie-Alix ROUSSILLON • Vincent LEGENDRE • Michel RIEU \\ Ifip - Institut du porc, Toulouse
}

La majorité des porcs sont commercialisés en France directement par les Organisations de producteurs. La fixation du prix est transparente et s'appuie sur les cotations du Marché au Cadran, deux fois par semaine. Contrairement à d'autres pays, les contrats à livraisons différées sont très peu développés en France sur ce marché, pour compenser les fluctuations du prix du porc. Toutefois, cette variabilité fragilise les opérateurs de la filière et une meilleure coordination constituerait une voie d'amélioration globale de la compétitivité.

MOTS-CLÉS : marché, prix, commercialisation, contractualisation, porcs

\begin{abstract}
Animal marketing contracts in France - an answer to hog price variability?
In France, most hogs are sold by Producer Organisations. The pricing is transparent and based on the Dutch auction of the Breton pig Market (MPB), twice a week. Unlike some other countries, marketing contracts aren't developed in France on this market, to compensate for the pork price fluctuations. However, this variability weakens the operators. Better coordination would be a way of improving overall competitiveness. (JEL: Q13)
\end{abstract}

KEYWORDS: price, pork, sales, marketing, contracts

A vec environ 24 millions de porcs élevés et abattus en 2013 (Baromètre porc, 2014) $)^{1}$, la France est le troisième producteur de l'Union européenne (UE).

Le soutien politique direct à la production porcine a toujours été très limité dans l'UE. Dès les débuts de la PAC a été choisie une organisation libérale du marché du porc, à la différence de celui d'autres grands marchés agricoles européens (lait, céréales, viande bovine...). Malgré cela, l'évolution de la PAC vers une politique moins interventionniste se traduit par un affaiblissement des instruments relatifs à la filière porcine.

- Des restitutions aux exportations ont par le passé compensé un écart de prix

1. Baromètre porc (2014). Supplément-Bilan 2013. Publication Ifip, $\mathrm{n}^{\circ} 441$, avril, p. 2. important entre le marché européen et le reste du monde et servi comme outil de gestion de crise. Elles n'ont plus été utilisées depuis 2008.

- Des aides au stockage privé peuvent aussi être accordées en cas de crise (Ifip, 2013 c) $)^{2}$. Lors de la crise de la dioxine qui a touché l'Allemagne en janvier 2011, les entreprises ont pu bénéficier de ce soutien et financer la congélation de l'excès d'offre qui ne trouvait pas de débouché.

- Sous forme de droits de douane, des barrières tarifaires protègent le marché communautaire des produits en provenance des pays tiers. Il existe cependant

2. Ifip (2013c). Le marché du porc : la France sur l'échiquier mondial. Fiche RMT «Économie des filières animales », 4 p., http://rmt-economiefilieres-animales.fr/. 
pour certains produits des contingents limités d'importation pour lesquels les droits de douane sont réduits ou nuls. Ils sont toutefois peu utilisés. Les barrières non tarifaires (statut sanitaire des troupeaux, agréments des abattoirs, interdiction des hormones de croissance comme la ractopamine) sont aussi très effectives.

Dans ce contexte libéral, la production porcine française s'est structurée autour des groupements de producteurs qui ont été à l'origine de son dynamisme dès les années 1970. Ce développement a été accompagné de la mise en place d'un mode original de fixation des prix : le marché au Cadran. Mais depuis les années 2000, alors que les concurrents allemands et espagnols connaissent un très fort développement, la production porcine française est en diminution, affaiblissant la dynamique de l'ensemble des maillons. En plus de leur volatilité, la forte hausse des cours des matières premières de l'alimentation porcine à partir de 2007 a lourdement pesé sur les coûts de production en élevage. La difficulté à répercuter ces hausses jusqu'au distributeur fragilise les maillons intermédiaires, abatteurs et transformateurs.

Dans un contexte économiquement difficile, la filière porcine française fait face à de nombreux défis, dont la variabilité du prix du porc.

Face à cette problématique, cet article se concentre sur les relations commerciales entre les maillons de la production et de l'abattage. Il présente le contexte et les acteurs de la commercialisation des porcs, puis décrit le mode de fixation du prix. Un dernier point propose une analyse sur les premières tentatives de contrats de commercialisation des porcs en France et présente brièvement les expériences étrangères. Une discussion ouvre des pistes de réflexion plus globales sur l'intérêt et la possibilité de mise en œuvre de contrats au sein de la filière porcine.

\section{Contexte et acteurs de la commercialisation des porcs}

En Europe, les porcs sont, dans une large mesure, produits selon des standards voisins sans différenciation marquée. Cela entraîne une concurrence généralisée qui oblige en permanence tous les producteurs à des efforts d'amélioration de leurs performances techniques et économiques afin de rester compétitifs.

\section{Une production organisée}

Les 24,4 millions de porcs élevés en France en 2013 marquent un recul de près de $10 \%$ par rapport au sommet de 1999. Plus de trois quarts de l'activité d'élevage et d'abattage sont concentrés dans un ensemble «Grand Ouest », comprenant les régions Bretagne, Pays de la Loire, PoitouCharentes et Basse-Normandie (Ifip, 2013a) $)^{3}$.

Au début des années 1970, les responsables professionnels du secteur et les pouvoirs publics ont fait ensemble le choix d'une production sans « intégration » (production pour tiers sous contrat), avec des éleveurs de porcs indépendants, mais organisés collectivement. Ces derniers achètent, vendent et assument directement l'essentiel des risques de marché. Dans leur grande majorité, ils appartiennent à des groupements de producteurs, ou Organisations de producteurs (OP) selon la dénomination choisie par les lois d'orientation agricole de 1996 et 2006 (Ifip, 2013b) $)^{4}$.

En 2012, 40 OP ont commercialisé 22 millions de porcs charcutiers, soit environ $90 \%$ de la production contrôlée en

3. Ifip (2013a). Le Porc par les chiffres. Paris, Édition 2012/2013, Ifip, 48 p.

4. Ifip (2013b). Organisation de la filière porcine française : de nouveaux équilibres économiques à trouver. Fiche RMT «Économie des filières animales », 6 p., http://rmt-economie-filieres-animales.fr/. 
Tableau 1. Situation des Organisations de producteurs en France

\begin{tabular}{lrrrr}
\hline & 1980 & 1990 & 2000 & \multicolumn{1}{c}{2012} \\
\hline Nombre d'OP & 204 & 145 & 92 & 40 \\
Nombre d'adhérents & 31500 & 23180 & 15100 & 10300 \\
Truies détenues (milliers) & 772 & 1004 & 1255 & 1100 \\
\hline
\end{tabular}

Source : IFIP d'après Coop de France.

Tableau 2. Groupements et porcs charcutiers commercialisés selon les ventes annuelles

\begin{tabular}{l|c|c|c|c}
\hline \multirow{2}{*}{ Ventes annuelles } & $\begin{array}{c}\text { Nombre } \\
\text { de groupements }\end{array}$ & $\begin{array}{c}\text { Part des porcs } \\
(\%)\end{array}$ & $\begin{array}{c}\text { Nombre } \\
\text { de groupements }\end{array}$ & $\begin{array}{c}\text { Part des porcs } \\
(\%)\end{array}$ \\
\cline { 2 - 5 } & \multicolumn{2}{|c|}{2002} & \multicolumn{2}{|c}{2012} \\
\hline + 3 millions & 1 & 13,1 & 2 & 40,6 \\
1 à 3 millions & 4 & 22,0 & 4 & 27,6 \\
500 000-1 million & 9 & 26,5 & 3 & 11,9 \\
$100-500000$ & 35 & 31,5 & 19 & 17,8 \\
Moins de 100 000 & 38 & 7,0 & 12 & 2,1 \\
Ensemble & 87 & 100,0 & 40 & 100,0 \\
\hline
\end{tabular}

Source : IFIP d'après Coop de France, guide Hors-Sol et données d'entreprises.

France. Le nombre d'OP a diminué dans le temps sous l'effet des mouvements de fusion. Il en existait 92 en 2000 et plus de 200 au début des années 1980 (tableau 1). Le nombre d'éleveurs adhérents a fortement baissé entre 1980 et 2010 (plus de $66 \%$ ) tout comme le nombre d'éleveurs de porcs en France (-50 \% pour les élevages de 100 porcs et plus).

Les six premières OP comptent chacune pour plus d'un million de porcs par an et rassemblent plus des deux tiers de la production nationale (tableau 2). À cellesci, s'ajoutent 3 OP gérant chacune entre 500000 et 1 million de porcs par an, représentant $12 \%$ de la production française. Les OP sont pour plus de $80 \%$ des coopératives ou unions de coopératives.

Progressivement, ces organisations ont développé des liens capitalistiques en amont (aliment, génétique) ou en aval (abattage, transformation, voire circuit de vente au détail) au sein de coopératives spécialisées ou polyvalentes.
Brousset et al. (1998) ${ }^{5}$ ont établi une typologie des filières porcines françaises coordonnées verticalement, en fonction du maillon à l'origine de la coordination ou central dans son fonctionnement. Selon un principe voisin, des types de filières ont été identifiés sur la base des capitaux détenus en 2012/2013 (figure 1). Ces organisations sont plus ou moins intégrées de l'amont à l'aval, à capitaux coopératifs ou privés et avec des liens financiers parfois complexes entre maillons. Faute d'informations assez précises, les parts dans la distribution n'ont pas été évaluées, mais certains types associent des distributeurs.

Environ un tiers de la production de porc et de l'abattage est issu de structures à capitaux coopératifs intégrant plus de trois

5. Brousset S., Rieu M., Vignau-Loustau L. (1998). Approche typologique du fonctionnement, de l'organisation et des orientations des groupements de producteurs de porcs de l'ouest de la France. Journées de la Recherche Porcine en France, vol. 30, pp. 153-159. 
Figure 1. Organisations au sein de la filière porcine

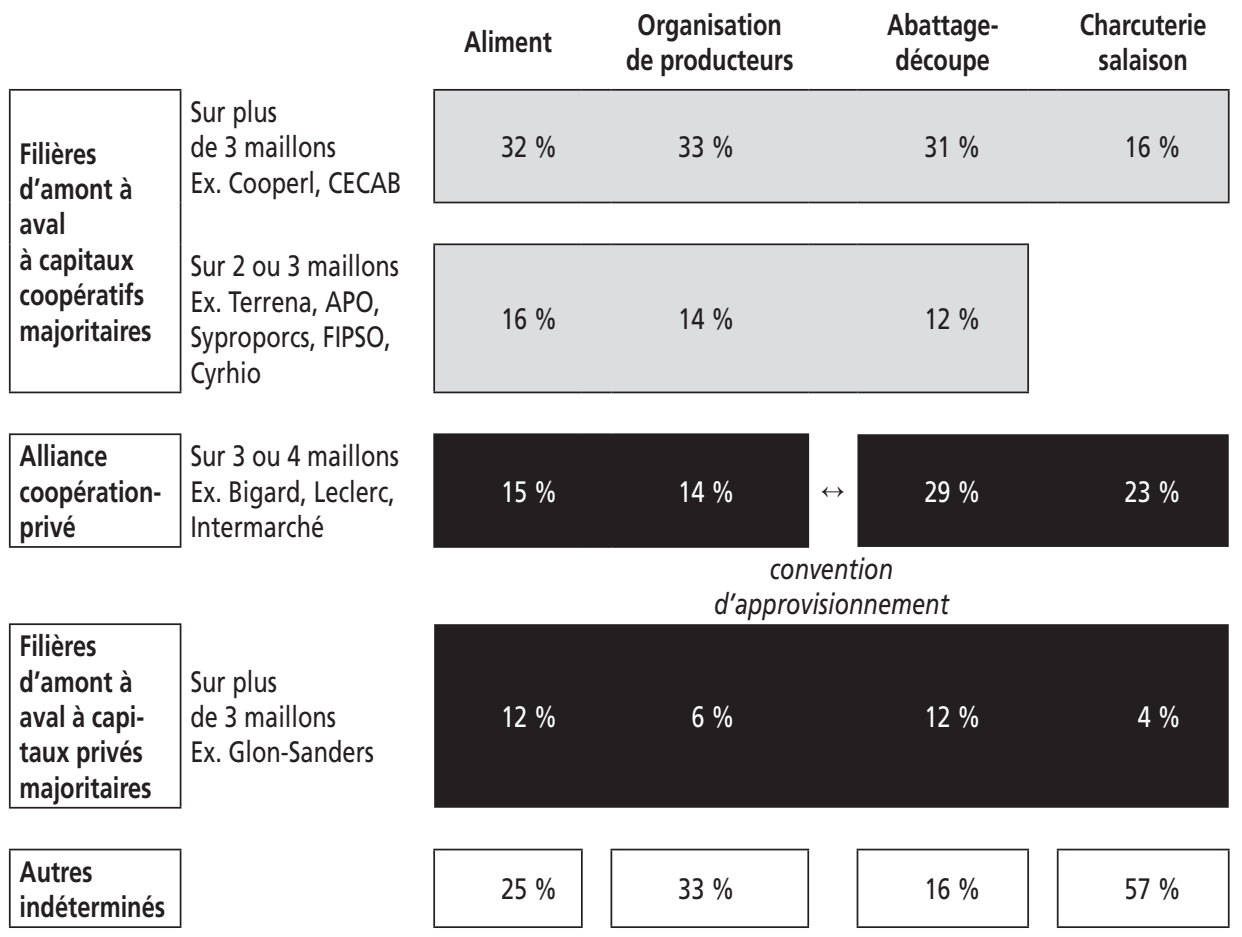

Note : Les pourcentages indiquent la part du type dans le total national de l'activité

\begin{tabular}{|l|l|}
\hline$X \%$ & Capitaux majoritairement coopératifs \\
\hline$X \%$ & Capitaux majoritairement privés \\
\hline$X \%$ & Capitaux privés comme coopératifs \\
\hline
\end{tabular} maillons de la filière. Ainsi la COOPERL, spécialisée dans la production porcine, intervient aujourd'hui le long de l'ensemble de la chaîne de valeur, de la génétique à l'abattage-découpe, dans la charcuterie-salaison et fait une expérience de vente au détail.

Dans d'autres coopératives, le nombre de maillons de la filière est plus réduit. Il peut aller de l'alimentation du bétail à l'abattage. C'est le cas de coopératives régionales hors Ouest Cyrhio, FIPSO et APO. En Bretagne, la coopérative Le Gouessant, spécialiste de l'aliment du bétail, a une participation dans la SICA Syproporcs, Organisation de producteurs.
Ce type de filière représente environ $14 \%$ de la production et $12 \%$ de l'abattage français de porcs.

Il existe également des filières où le jeu des participations mêle des structures coopératives et privées (15\% de la production et $22 \%$ des abattages). Une seule filière conséquente est pilotée par une structure à capitaux privés majoritaires, avec la firme d'alimentation animale Glon-Sanders. Quelque $30 \%$ de la production et de l'alimentation animale, $20 \%$ des abattages et près de $60 \%$ de la charcuterie-salaison sont issus d'entreprises sans coordination au sein de la filière, au moyen de partage de capital, ou de taille trop modeste pour être visibles. 
Tableau 3. Principales entreprises d'abattage de porcs

\begin{tabular}{lclc}
\hline \multicolumn{1}{c}{ Groupe } & Porcs abattus (milliers) & Type d'opérateur & $\begin{array}{c}\text { Nombre } \\
\text { d'abattoirs }\end{array}$ \\
\hline Cooperl Arc Atlantique & 4886 & Coopérative & 3 \\
Bigard - Socopa Viandes & 4657 & Privé & 7 \\
Gad (Cecab-Prestor)* & 2362 & Coopérative & $2^{*}$ \\
Kermené & 1815 & Privé & 1 \\
Jean Floc'h & 1761 & Privé & 2 \\
Gâtine Viandes & 1117 & Privé & 1 \\
Tradival & 1096 & Coopérative & 2 \\
Abera & 1009 & Privé & 1 \\
AlM & 771 & Privé & 1 \\
FIPSO Industrie & 610 & Coopérative & 2 \\
Top 10 & 20084 & & \\
Abattages nationaux & 24340 & & \\
\hline
\end{tabular}

Notes : Activité 2012 consolidée ; *en octobre 2013, l'abattoir Gad de Lampaul a été fermé, l'activité transférée sur le second site du groupe à Josselin. En septembre 2014, la liquidation judiciaire de Gad a été prononcée.

Source : IFIP, selon UNIPORC et entreprises.

\section{L'abattage-découpe Des opérateurs privés ou coopératifs}

Certaines entreprises détiennent plusieurs abattoirs ou sites d'abattage (tableau 3). En 2012, les dix premiers groupes d'abattage comptent pour plus de $80 \%$ de l'activité nationale (Ifip, 2013a).

Quatre coopératives se placent dans le top 10 des abatteurs français. Certains groupes d'abattage ont des organisations complexes, avec une limite floue entre parties coopérative et privée : coopératives avec des filiales de statut privé ou groupes privés avec des coopératives parmi leurs actionnaires.

L'approvisionnement des abattoirs se fait de façon quasi exclusive par le biais des OP, et très rarement directement par le producteur ou via des négociants privés.

On compte 32 abattoirs de plus de 100000 porcs/an, réalisant $90 \%$ de l'activité nationale (données de 2012). Parmi eux, dix sites d'abattage de plus de un million de porcs/an (voir tableau 4) assurent $57 \%$ du total. Le nombre total d'abattoirs a chuté de 424 à 254 entre 1990 et 2000.

Tableau 4. Abattoirs français de porcs selon la classe de taille (évolution)

\begin{tabular}{l|cc|cc|cc}
\hline \multirow{2}{*}{$\begin{array}{c}\text { Porcs abattus } \\
\text { par abattoir et par an }\end{array}$} & \multicolumn{2}{|c|}{1990} & \multicolumn{2}{c|}{2000} & \multicolumn{2}{c}{2012} \\
\cline { 2 - 7 } & $\begin{array}{c}\text { Nombre } \\
\text { d'abattoirs }\end{array}$ & $\begin{array}{c}\text { Part de } \\
\text { l'activité } \\
\text { nationale }\end{array}$ & $\begin{array}{c}\text { Nombre } \\
\text { d'abattoirs }\end{array}$ & $\begin{array}{c}\text { Part de } \\
\text { l'activité } \\
\text { nationale }\end{array}$ & $\begin{array}{c}\text { Nombre } \\
\text { d'abattoirs }\end{array}$ & $\begin{array}{c}\text { Part de } \\
\text { l'activité } \\
\text { nationale }\end{array}$ \\
\hline Plus de 1 million & 3 & 18 & 10 & 56 & 10 & 57 \\
De 500000 à 1 million & 9 & 34 & 3 & 8 & 7 & 20 \\
De 100000 à 500 000 & 22 & 23 & 29 & 22 & 15 & 15 \\
Moins de 100 000 & 390 & 25 & 212 & 14 & 141 & 7 \\
Total & 424 & 100 & 254 & 100 & 173 & 100 \\
\hline
\end{tabular}


La concentration s'est ralentie après 2000, puisqu'il y avait 173 abattoirs en 2012.

\section{Méthode de fixation des prix}

\section{Un prix de référence}

Avec de premières initiatives en 1972 et la création du Marché du porc breton (MPB) (marché au cadran) en 1975, les éleveurs ont voulu rééquilibrer le rapport de force avec les abatteurs et rendre transparente la fixation du prix.

La cotation est établie le lundi et le jeudi de chaque semaine (Ifip, 2013b). Une convention commerciale interprofessionnelle fixe des règles détaillées de fonctionnement du marché, comme le plafonnement des variations des prix, les conditions de paiement des porcs, avec un prix de base, départ ferme, hors TVA, pour une qualité donnée et un délai de paiement maximal (14 jours).

Environ 65000 porcs par semaine en moyenne, soit de l'ordre de $13 \%$ du total hebdomadaire abattu en France, sont commercialisés via le MPB. L'origine géographique très concentrée des animaux (en majorité le Finistère, puis les Côtesd'Armor) et le faible nombre des acheteurs (Bretagne et Grand Ouest) limite la confrontation offre/demande à la Bretagne. Malgré cela, ce marché a une importance déterminante dans le fonctionnement de la filière et un rôle directeur avéré dans la formation du prix du porc en France, quel que soit le statut (coopératif ou privé) de l'abattoir (Ilari et al., 2009) ${ }^{6}$.

La convention du MPB fixe une grille de rémunération en fonction du poids et de la qualité des carcasses, appréciée selon un critère clairement établi, le Taux de muscle

6. Ilari E., Duflot B.,Van Ferneij J.-P., Marouby H., Rieu M. (2009). Le prix du porc perçu par les producteurs dans l'Union européenne. Les études économiques de l'Ifip, $81 \mathrm{p}$. des pièces (TMP) (Daumas, 2006) ${ }^{7}$, sous forme de plus ou moins-values. La plupart des abattoirs appliquent la même grille de qualité, mais pour les grilles de paiement au poids, il existe plus de différences régionales, principalement pour prendre en compte la production de porcs lourds. Ces grilles proviennent la plupart du temps des organisations interprofessionnelles, mais peuvent aussi être issues des abattoirs. Les porcs sont pesés et classés par des associations interprofessionnelles de type syndical. Uniporc Ouest garantit les opérations de pesée et de classement des porcs du Grand Ouest et du Nord de la France, soit $85 \%$ des abattages nationaux.

En France, la quasi-totalité des paiements des porcs se fait sur la base du prix au «Cadran » (moyenne d'une ou plusieurs séances) auquel s'ajoutent les diverses plus-values déterminées par les grilles évoquées ci-dessus, ou divers cahiers des charges (traçabilité, certification, IGP, Label Rouge...).

Ce système, avec ses grilles de paiement, a un effet incitatif indéniable. Les éleveurs et leur amont adaptent leurs pratiques à tout changement de grille de poids et de TMP pour obtenir le meilleur paiement possible des porcs. Nécessaire dans les années 1970 pour rééquilibrer le rapport de force en faveur des éleveurs, cette farouche volonté de rationalisation de la production et de rassemblement a pu, par la suite, apparaître comme un « dogme » de standardisation dans une stratégie exclusive de compétitivité par les coûts, négligeant la diversification pour répondre à des besoins plus variés.

\section{Formation du prix du porc}

Le prix du porc est très variable en France, comme dans l'ensemble de l'UE et chacun des pays membres (figure 2).

7. Daumas G. (2006). Les nouvelles modalités de classement des porcs. Techniporc, vol. 29, $\mathrm{n}^{\circ} 6$, pp. 23-26. 
Figure 2. Évolution du prix au Cadran en France, de 2000 à 2013

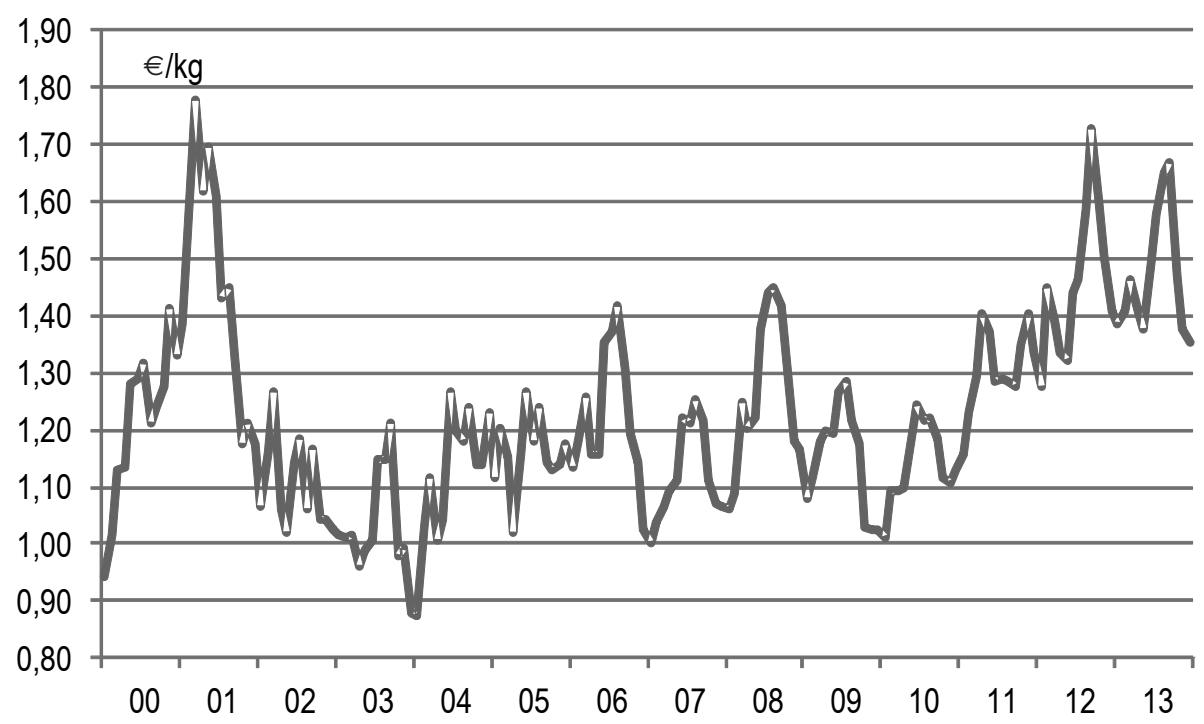

Note : Moyennes mensuelles.

Source : MPB.

Figure 3. Évolution des composantes du prix du porc en France

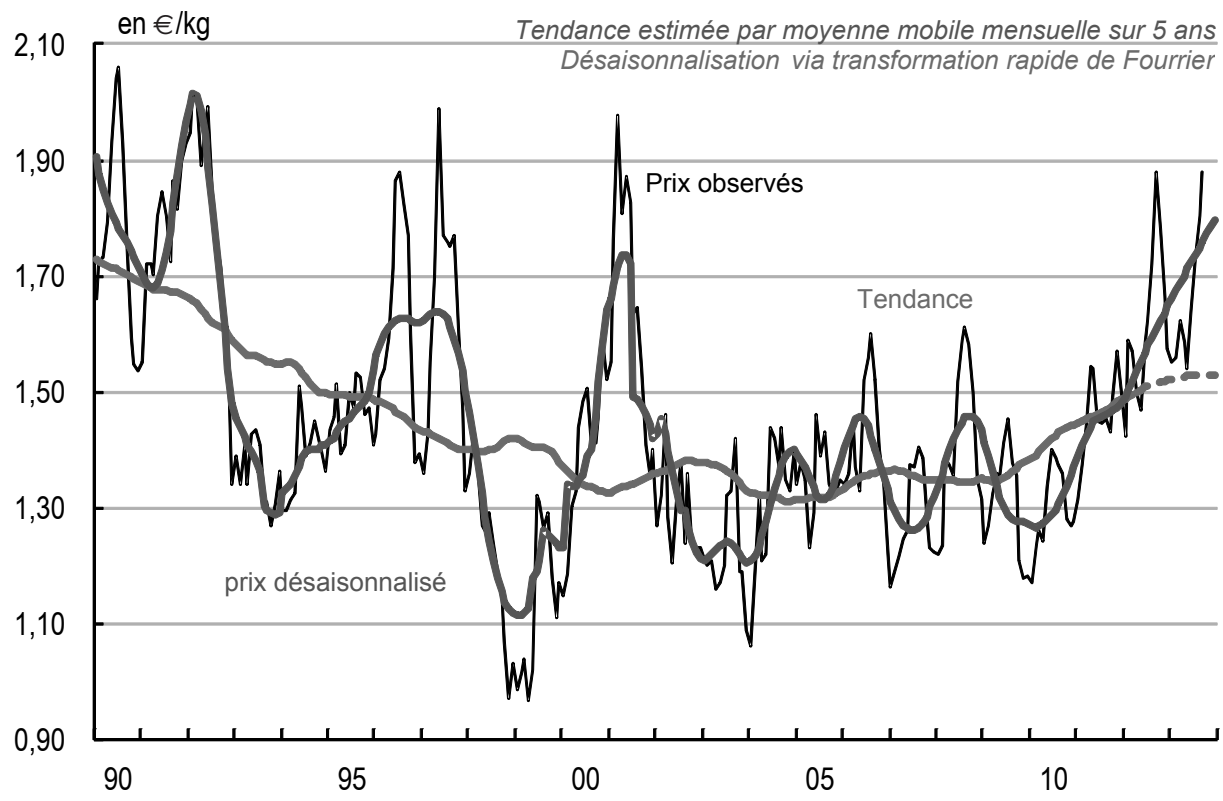

Note : prix d'achat par les abattoirs, cotation nationale de qualité classe $\mathrm{E}$, en $€ \mathrm{~kg}$ de carcasse.

Source : calculs Ifip, d'après données FranceAgriMer. 
Il évolue selon plusieurs « composantes » correspondant à différents horizons de temps, représentées sur la figure 3.

- Tendance. À long terme, le prix du porc est principalement déterminé par le coût de production. Il se raisonne au niveau de la zone UE du fait d'importants échanges au sein d'un marché ouvert. En baisse depuis 1990 sous l'effet du recul du coût de l'aliment, la tendance s'est stabilisée durant les années 2000 puis a augmenté à la fin de la décennie, avec le fort redressement des prix de l'alimentation animale (estimations de mars 2012 à décembre 2013).

- Cycle. À moyen terme, le « cycle » alterne régulièrement des hausses et des baisses de prix, en opposition avec celles, beaucoup moins amples, de la production porcine européenne. De deux ans environ dans les années 1970 et 80 (deux fois le cycle biologique de production, à savoir de l'insémination des truies à l'abattage des porcs), il s'est allongé à cinq ans à partir des années 1990. Il s'agit d'un mécanisme d'adaptation de l'offre à la demande de l'UE régulée par le prix. Depuis plusieurs années, des rigidités structurelles réduisent l'adaptabilité de l'offre aux variations du prix (« cycle »). Les élevages sont plus spécialisés et réagissent moins vite aux fluctuations du cours du porc. Par ailleurs, les règles environnementales ont contingenté la production, par des quotas de droit ou de fait (Pays-Bas, Bretagne...). Au début des années 2000, les variations cycliques de la production européenne (UE-15) s'étaient atténuées. Mais l'élargissement aux pays d'Europe centrale, qui ont encore de nombreux petits détenteurs de porcs (Pologne, Hongrie...), leur a redonné une certaine vigueur.

- Saisonnalité. Par définition, la saison se répète à l'identique chaque année. Cependant, des changements peuvent apparaître sur une longue période. La saisonnalité (évolution intra-annuelle) du prix du porc dépend de la saisonnalité de l'offre (prolificité, poids des porcs) et de celle de la demande (consommation spécifique des pièces). Le porc est mieux valorisé en été, quand l'offre est réduite (pour des raisons physiologiques) et la demande forte (barbecue, sandwich...) qu'en hiver.

- Aléas. Des chocs peuvent affecter l'offre ou la demande en porcs. Divers en ampleur et en nature, ils ont des impacts variés, mais pèsent de plus en plus sur la formation du prix du porc en Europe. Ils peuvent revêtir une ampleur telle qu'ils masquent ou contrecarrent les mécanismes présentés plus haut. La participation plus active au marché mondial des pays de l'UE fait de plus en plus intervenir des facteurs extérieurs : politiques (unification allemande en 1990, élargissements de l'UE) sanitaires (peste porcine aux Pays-Bas en 1997, ESB et reports de consommation en 1996 et $2001 \ldots$ ), économiques (crise mondiale de 2008, flambées du prix des céréales, fermeture soudaine des marchés pour des raisons sanitaires et parfois politiques). Ils affectent l'offre, la demande ou la rentabilité des élevages et des opérateurs industriels, et perturbent les ajustements (Roussillon, 2013). ${ }^{8}$

Le « cycle du porc », connu et prévisible par le passé, a accompagné et stimulé la restructuration de la production européenne dans les années 1980-1990. Aujourd'hui, des facteurs extérieurs viennent perturber cette logique, qui devient moins perceptible.

8. Roussillon M.A. (2013). L'équilibre des prix dans la filière porcine : vers un scénario de rupture ? Techporc, $\mathrm{n}^{\circ} 14$, pp. 2-4. 


\section{Les contrats de commercialisation des porcs et produits du porc}

En agriculture, les contrats sont principalement utilisés pour garantir un débouché, un approvisionnement, une qualité de produit, une marge ou un prix (BouamraMechemache et al., 2015)9. Différents contrats peuvent impliquer les producteurs de porcs en France : achat d'aliment à un fabricant, transactions de porcelets entre naisseurs et engraisseurs ou engraissement dit « à façon » de porcs par des sous-traitants (Roguet et al., 2008) ${ }^{10}$. L'adhésion des éleveurs à un groupement est formalisée par un bulletin d'engagement, un contrat particulier précisant un niveau d'approvisionnement en porcs charcutiers, une durée et un mode de rémunération des porcs, basé sur le prix du MPB. Tous les groupements fonctionnent sur ce modèle (communication de Coop de France en 2013). Il existe des démarches de prix garantis pour des éleveurs dans des situations particulières (investissements pour développer significativement le naissage, installations hors cadre familial...), financées par l'effort collectif du groupement. Elles sont rares. La plupart des coopératives mettent plutôt en place un système d'accompagnement, tant pour les démarches administratives que pour le suivi technicoéconomique.

Entre groupements et abatteurs, il existe peu de contrats écrits. La vente repose le plus souvent sur une pratique habituelle ou sur une entente valant contrat.

9. Bouamra-Mechemache Z., DuvaleixTréguer S., Ridier A. (dans ce numéro) (2015). Contrats et modes de coordination en agriculture. Économie rurale, $\mathrm{n}^{\circ} 345$, Janvier-février.

10. Roguet C., Laugé V., Rieu M. (2008). La production et les flux de porcelets en France. Les études économiques de l'Ifip, $61 \mathrm{p}$.

\section{Les premières tentatives de contrats en France}

\section{Initiative Syproporcs-Kerméné}

En mars 2011, une initiative entre l'Organisation de producteurs bretonne Syproporcs et l'abatteur Kerméné, basé dans les Côtes-d'Armor (groupe Édouard Leclerc), permet de commercialiser en moyenne 400 porcs par semaine, via des contrats à livraison différée (CLD) de 14 semaines environ (tableau 5).

Il s'agit d'un contrat d'engagement tripartite entre l'éleveur, le groupement Syproporcs et l'abatteur, en l'occurrence Kerméné. Le groupement et l'abattoir ont signé un contrat-cadre précisant les engagements de chacun, comme le rôle de garantie du groupement en cas de défaut de livraison. L'éleveur signe ensuite un engagement par lot vendu via le CLD, précisant le volume, le prix et la date de livraison des porcs.

Près de $10 \%$ des adhérents sont des utilisateurs réguliers des CLD. Les lots sont de taille variable, le plus souvent entre 50 et 200 porcs.

L'abatteur propose chaque semaine un prix à cinq semaines ou plus (14 semaines maximum). Cette cotation est indiquée aux adhérents de Syproporcs par lettre hebdomadaire. Ils sont libres de prendre ou non une position sur cette vente à terme.

Certains vendent une part variable de leur production (30 à $50 \%$ le plus souvent)

Tableau 5. Évolution des signatures de contrats à livraison différée de porcs entre les adhérents de I'OP Syproporcs et l'abatteur Kerméné

\begin{tabular}{lcc}
\hline & $\begin{array}{c}2011 \\
\text { (mars-déc.) }\end{array}$ & 2012 \\
\hline Nombre d'éleveurs & 32 & 37 \\
\hline $\begin{array}{l}\text { Nombre de contrats } \\
\text { passés }\end{array}$ & 17290 & 20000 \\
\hline $\begin{array}{l}\text { Nombre de porcs } \\
\text { commercialisés }\end{array}$ & & 96 \\
\hline
\end{tabular}

Source : Syproporcs. 
par CLD et le reste classiquement par le Cadran, selon une formule proposée par Syproporcs. Elle permet à l'éleveur de jouer sur deux tableaux, en se confrontant pour partie aux fluctuations des prix spot, qui sont négociés sur l'instant et qui traduisent l'état de l'offre et de la demande au moment de la vente et, pour une autre partie, en fixant son prix de vente. L'utilisation du CLD suppose toutefois une bonne connaissance par l'éleveur de ses prix de revient ; c'est pour cela que tout éleveur s'engageant dans cette démarche est tenu de suivre une formation en ce sens. Par ailleurs, l'éleveur peut sécuriser une partie de ses achats, notamment en passant des contrats sur ses approvisionnements en aliment, qui représente 60 à $70 \%$ de son coût de revient total.

Du côté des abatteurs, Kerméné affiche un objectif de sécuriser une partie de ses approvisionnements, notamment lorsque l'offre est réduite par rapport à la demande et que la lutte est la plus rude sur le Marché du porc breton (Poilvet, 2011) ${ }^{11}$. C'est aussi une assurance pour l'avenir, en anticipant une réduction de l'offre régionale et nationale. Cela lui permet aussi d'être précurseur dans la démarche.

\section{Le contrat à livraison différée}

\section{du Marché du porc breton}

Après cette initiative de Syproporcs, le MPB a proposé, fin 2011, un CLD de 6 à 20 semaines, ouvert à tout éleveur adhérent d'un groupement de producteurs cotisant au MPB. Trois acheteurs se sont enregistrés : l'abattoir Abera, entreprise du groupe Glon, le groupe Cooperl et l'abattoir Kerméné. Dans les faits, seul ce dernier a acheté des porcs via les contrats à livraison différée du MPB, en plus des achats réalisés via d'autres contrats avec Syproporcs (cf. supra).

11. Poilvet D. (2011). La vente à livraison différée arrive au Marché du porc breton. Réussir Porc, $\mathrm{n}^{\circ} 187$, pp. 6-7.
La vente à livraison différée au MPB est encadrée par une convention signée par le MPB et chacun des acteurs présents, tant acheteurs qu'organisation de producteurs (OP). L'éleveur fait parvenir au MPB un bulletin d'annonce où il précise le nombre total de porcs proposés (par lots de 50), la semaine de livraison et un prix d'objectif, qui ne paraît pas dans le catalogue. Ce bulletin d'annonce est cosigné par l'OP, qui s'engage à être solidaire de ses adhérents pour l'exécution de leur engagement.

La vente est réalisée par internet. Si le prix d'objectif de l'éleveur n'est pas atteint, les propositions de prix des acheteurs lui seront transmises. L'éleveur a la possibilité de refuser la vente et de la reporter à la prochaine séance.

Lors des premiers mois suivant la mise en œuvre du CLD du Marché du porc breton, le nombre de porcs présentés a oscillé entre 400 et 5000 têtes par semaine, pour fortement ralentir par la suite. Malgré des écarts de prix ponctuellement importants, il faut noter qu'il n'y a eu aucun défaut de livraison de la part des éleveurs. En 2013, aucun porc n'a été commercialisé par ce biais ; les dernières ventes datent de décembre 2012.

\section{Exemples à l'étranger}

\section{Dans l'Union européenne}

La commercialisation des porcs sur la base d'un prix spot constitue la pratique actuellement la plus répandue en Europe. Les alternatives sont rares ; le dernier marché à terme, basé à Hanovre puis maintenant à Francfort (Eurex), peine à fonctionner. Une étude de l'IFIP (Roguet et Rieu, 2004) $)^{12}$ a mis en évidence les limites de ce type d'outil pour la production porcine européenne.

12. Roguet C., Rieu M. (2004). Le marché à terme pour gérer le risque de prix du porc : mythe ou réalité ? Techniporc, vol. 27, n 5, pp. 3-9. 
Dans certains pays, les contrats de production prédominent, comme en Belgique ou en Espagne. La moitié de la production espagnole serait détenue dans le cadre de contrats d'intégration et $70 \%$ en Catalogne (selon la fédération des coopératives agricoles de Catalogne en 2009). L'intégrateur est une société qui généralement fabrique des aliments composés et possède des participations financières dans un abattoir. Elle est propriétaire des animaux et l'éleveur du bâtiment, le plus souvent de grande taille (entre 500 et 3000 truies ou plus de 2000 porcs à l'engrais). L'éleveur reçoit une rémunération fixe, pour un cycle de production ou la durée du contrat.

En Angleterre et en Italie, 80 à $90 \%$ des animaux sont vendus via des contrats de moyenne à longue durée. Les groupements de producteurs sont rares et il s'agit souvent pour les éleveurs de garantir un débouché pour leurs animaux. En Italie, les contrats sont d'une durée d'un an, basés sur le prix de référence national, avec le plus souvent un bonus (volume livré, durée du contrat, production spécifique...). Au Royaume-Uni, les contrats durent de deux à cinq ans, avec des préavis de rupture de trois à six mois. La livraison est le plus souvent hebdomadaire, pour un nombre de porcs déterminé, aux caractéristiques précises. La constance des volumes livrés, le plus souvent à un rythme hebdomadaire, est récompensée par un bonus. La valorisation des porcs se base sur un prix à fixation hebdomadaire déterminé dans le contrat. Il existe de nombreuses références de prix en Angleterre (spot, industrie, officiel...), qui permettent aux contrats de s'adapter aux besoins diversifiés de l'aval (porcs plus ou moins lourds, plus ou moins gras...).

Au Danemark, près de $90 \%$ des porcs abattus le sont dans des coopératives auxquelles adhèrent les éleveurs. Ils sont rémunérés par un prix spot fixé par la coopérative (Danish Crown ou Tican), complété en fin d'année par un bonus en fonction des résultats de l'entreprise. Aux Pays-Bas le commerce des porcs est organisé sur un mode libéral, où l'éleveur effectue la mise en marché de ses porcs. En Allemagne, les contrats sont rares, représentant souvent moins de $5 \%$ de la production. Lorsqu'ils existent, ils ont surtout pour but de fidéliser des producteurs de filières spécifiques en proposant des bonus par rapport au prix de marché (Antoine et Marouby, 2013) ${ }^{13}$.

\section{Aux États-Unis}

Selon une étude réalisée en 2007 et actualisée en 2013 (non encore disponible), Key et $\mathrm{McBride}^{14}$ estiment que quelque $70 \%$ des porcs aux États-Unis, issus de $30 \%$ des élevages du pays, sont sous contrat de production (production contract). C'est particulièrement le cas pour les élevages de types naisseur ou engraisseur. Cette organisation a permis de développer la taille des élevages aux États-Unis. La plupart des intégrateurs commercialisent les porcs produits par leurs sous-traitants via des contrats de commercialisation ou marketing contracts (MacDonald et Korb, 2011) ${ }^{15}$.

Les différents modes de commercialisation des porcs sont recensés grâce au Livestock Mandatory Price Reporting Act de 1999, qui impose le suivi quotidien des prix et volumes commercialisés. Globalement, la part de la production commercialisée par contrat est en forte croissance depuis le début des années 1990 (Lawrence et al., 2007) ${ }^{16}$.

13. Antoine E., Marouby M. (2013). Fixer le prix du porc par contrat : expériences à l'étranger et enseignements pour la France. Les études économiques de l'Ifip, 62 p.

14. Key N., McBride W. (2007). The Changing Economics of U.S. Hog Production. USDA, Economic Research Report Number 52, 38 p.

15. MacDonald J. M., Korb J. (2011). Agricultural Contracting Update: Contracts in 2008. USDA ERS, Economic Information Bulletin, $\mathrm{n}^{\circ} 72,35 \mathrm{p}$. 16. Lawrence J. D., Hayenga M., Kliebenstein J., Rhodes V. J. (2007). Producing and marketing hogs under contracts. Fact Sheet, Pork Information Gateway, 11-03-07, 6 p. 
Les contrats de commercialisation sont le plus souvent des contrats dits à livraison différée (exécution ultérieure de termes décidés à la signature du contrat). Ils représentent $61 \%$ des porcs abattus dans le pays (Plain, 2013) ${ }^{17}$. Il en existe trois grands types :

Les market formula (39 \% des porcs commercialisés), contrats basés sur une cotation publique et contemporaine de la date de vente. Ils garantissent une livraison et un prix de marché. Ils sont appréciés des éleveurs dans les zones de moindre densité porcine, car ils limitent l'effet négatif de la localisation de l'éleveur en liant le contrat à une référence issue d'une zone de production importante.

Les other market formula (7\%), contrats basés sur le Marché à terme (MAT) de Chicago. L'abatteur garantit au producteur un prix de vente basé sur celui du MAT à la période de livraison choisie, cotée au jour de la signature du contrat. Cela permet à l'éleveur de connaître son prix et gérer sa marge, tout en prenant des décisions lot par lot. Ces contrats permettent aux partenaires de profiter des possibilités offertes par le MAT pour prendre des contrats « futures » sur une position opposée à celle du CLD. Cependant, cela nécessite de disposer de moyens financiers importants, pour s'acquitter des garanties nécessaires.

Ces deux grands types de contrats ne permettent pas un lissage du prix de vente des porcs.

Les other purchase agreement (15\%) regroupent des contrats avec d'autres modes de rémunération. Certains se basent sur la valeur reconstituée de la carcasse à partir des prix de gros des pièces, le plus souvent la cotation officielle de l'USDA. D'autres s'appuient sur une estimation du coût de production, ce qui garantit

17. Plain R. (2013). U.S. Market Hog Sales, 20022012. Agricultural Electronic Bulletin Board, University of Missouri Extension, $6 \mathrm{p}$. une marge à l'éleveur. Des systèmes de contrats à seuils ou à fenêtre encadrent le prix de référence pour protéger les partenaires du contrat de fortes fluctuations du prix du porc, à la hausse comme à la baisse (MacDonald et Korb, 2011, Antoine et Marouby, 2013).

\section{Discussion}

\section{Des contrats de commercialisation des animaux peu développés}

La prédominance du système naisseur-engraisseur en France, basé sur la conduite en bande des truies, a pour conséquence une commercialisation fréquente des porcs (de 1 fois par semaine à au moins 1 fois par mois), ce qui permet un lissage des prix à court ou moyen terme et diminue l'intérêt d'un contrat à prix fixe ou lissé sur un laps de temps similaire. La standardisation des pratiques et des produits rend les porcs vendus facilement interchangeables. La concentration géographique des acteurs et l'organisation de la filière porcine française limitent le risque de débouchés pour les éleveurs et d'approvisionnement pour les abatteurs. Le marché au cadran offre aux éleveurs une mode de fixation du prix clair et transparent. Cette référence bihebdomadaire est structurante pour le marché du porc en France. Jusqu'à présent, c'est plutôt au sein des filières différenciées à volumes de production restreints et à opérateurs peu nombreux, comme le porc biologique, que les opérateurs ont ressenti le besoin de contractualiser leurs échanges.

Cependant, les variations interannuelles des prix du porc et de l'aliment sont importantes, surtout pour l'aliment ces dernières années. Pour lisser le prix de vente des animaux et de l'aliment, des contrats de longue durée à prix fixés sont envisageables, ce qui n'est pas le cas actuellement, les contrats en porc ne dépassant pas les trois mois, limitant de facto leur intérêt. Il est cependant difficile de mesurer 
précisément les effets négatifs de la volatilité des prix sur les résultats économiques. De plus, au vu des coûts potentiels de mise en œuvre des contrats, des simulations précises seraient nécessaires pour comparer leur intérêt à celui d'autres dispositifs (épargne mutualisée, provisions défiscalisées...).

Toutefois, la contractualisation pourrait permettre à la filière porcine française de poursuivre d'autres objectifs pour compenser le manque de rentabilité économique de ses maillons :

- mieux adapter l'offre à la demande ;

- valoriser de manière plus optimale l'ensemble de la carcasse de porc ;

- inciter à produire exactement les différents types de porcs correspondant aux différents segments de la demande.

\section{Diversité des structures et pouvoirs de marché}

À l'aval de la filière porcine, dans le secteur de la charcuterie-salaison, les écarts de structures sont importants entre les opérateurs, avec des entreprises d'envergure européenne, voire mondiale, qui opèrent à côté de PME régionales. Les degrés de concentration divers des opérateurs génèrent des différences parfois importantes dans les pouvoirs de négociation. Cela se traduit entre autres par des difficultés à transmettre les variations de prix du porc le long de la filière jusqu'au consommateur.

Débouché majoritaire des produits de porc avec plus de $70 \%$ des tonnages, la grande distribution a un impact déterminant sur l'activité de la filière porcine. Le secteur est très concentré, six groupes se partageant près de $85 \%$ du marché des produits alimentaires (Leclerc, Auchan, Carrefour, Intermarché, Casino, Système U).

Les négociations à l'échelle nationale ou régionale occupant une place importante, la distribution dispose d'un fort pouvoir de marché face à ses fournisseurs, charcutiers/salaisonniers pour les produits transformés ou bien abatteurs-découpeurs dans le cas des produits frais. Dans un contexte de difficultés économiques impactant fortement le pouvoir d'achat des ménages, la concurrence entre distributeurs sur les prix de détail est particulièrement vive et accroît les difficultés de la filière à répercuter des hausses jusqu'au consommateur final. Des relations « historiques » lient les distributeurs aux industriels qui les approvisionnent, en particulier sur la viande fraîche de porc. Les fournisseurs varient relativement peu d'une année sur l'autre. Pourtant les négociations tarifaires sur la viande fraîche sont fréquentes, au rythme du marché au Cadran. Celles sur les produits de charcuterie sont établies sur des périodes plus longues.

\section{La variabilité des prix nuit à la coordination entre les opérateurs}

Les renégociations fréquentes liées à la variabilité du prix du porc au Cadran limitent les relations commerciales à des échanges de court terme, au détriment de stratégies de plus long terme entre les abatteurs et leurs clients, salaisonniers ou distributeurs.

Les abatteurs subissent les fluctuations hebdomadaires du prix du porc, mais aussi de la viande en fonction des besoins des clients (Van Ferneij et al., 2004) ${ }^{18}$. Ils ont à valoriser la carcasse par la vente d'une multitude de produits dont les prix diffèrent selon le temps et les lieux en fonction de l'offre et de la demande, à court, moyen ou long terme (Antoine-Ilari, 2011) ${ }^{19}$.

18. Van Ferneij J.-P., Rieu M., Vermeil de Conchard R. (2004). Le commerce de viande de porc issue de la découpe en France. Les études économiques de l'ITP, $101 \mathrm{p}$

19. Antoine-Ilari E. (2011). Caractéristiques et formation du prix des pièces de porc en France: Modélisation et prévision. Les études économiques de l'Ifip, $74 \mathrm{p}$. 
Malgré l'existence d'une interprofession nationale (Inaporc), la coordination entre les opérateurs de l'aval de la filière porcine est insuffisante, réduisant l'efficacité de la chaîne d'approvisionnement des charcutiers-salaisonniers ou des distributeurs et accroissant les coûts de transaction. Dans le souci de mieux répondre à la demande finale, les concertations entre fournisseur et client devraient être plus étroites et intégrer plus de spécifications sur le produit échangé.

Par ailleurs, une vision de plus long terme de l'offre et la demande, avec des prix d'échange fixés sur des périodes plus longues, permettrait des stratégies d'investissements, condition essentielle pour l'amélioration de la compétitivité des structures et un retour à la rentabilité. La contractualisation à terme des échanges y contribuerait (Roussillon, 2015) ${ }^{20}$.

\section{Des initiatives de contractualisation portées par l'aval de la filière}

La contractualisation s'est cependant développée dans des situations précises, notamment les filières différenciées (produits sous signes de qualité). Dans la filière porcine biologique, l'enseigne de distribution Système U a signé, début 2013, un partenariat avec près de 180 éleveurs de porcs bio, en collaboration avec des sociétés industrielles pour l'abattage-découpe et la fabrication d'une gamme de produits de porc bio frais et transformés (Legendre et al., 2013 $)^{21}$. Il s'agit pour l'enseigne de garantir son approvisionnement régulier et de qualité et d'accompagner le développement

20. Roussillon M.A., Legendre V., Rieu M. (à paraître) (2015). Adaptation de l'offre à la demande des produits du porc en France. Quelles voies d'action pour les opérateurs de l'aval de la filière ? Journées de la Recherche Porcine, vol. 47.

21. Legendre V., Cresson C., avec la participation d'O. Chapolard (2013). Marché et consommation du porc bio français. AlterAgri, $\mathrm{n}^{\circ} 119$, mai-juin, pp. 28-31. de la filière face à une offre en porc biologique parfois jugée insuffisante.

En février 2014, Système U et le groupe coopératif Terrena ont annoncé un engagement portant sur l'achat de 50000 tonnes de porc par an pendant trois ans, selon un contrat garantissant aux producteurs une plus-value de 8 à 12 centimes d'euro/kg de viande. La viande sera commercialisée sous la marque du distributeur avec la bannière « Porc Nouvelle Agriculture », respectant un cahier des charges portant des garanties sur l'alimentation et le bien-être animal. D'autres enseignes de la distribution annoncent leur volonté de passer des contrats avec l'amont de la filière porcine.

La chaîne de restauration rapide McDonald's poursuit également en France une politique de contractualisation sur ses différents approvisionnements (céréales, légumes...) avec des engagements sur les volumes et des prix fixés sur plusieurs années. Cela permet à l'enseigne de sécuriser une partie de ses approvisionnements. Des contrats tripartites avec producteurs et industriels se développent sur la viande bovine, mais les volumes concernés restent assez faibles, environ $3 \%$ des besoins de l'enseigne pour cette viande en 2012 (Agra Alimentation, 2013) ${ }^{22}$. En ce qui concerne son approvisionnement en jambon, un contrat renouvelé en juin 2014 lie l'enseigne de fast-food à un abatteur, un transformateur et à l'association « Cochon de Bretagne ». Le contrat prévoit la possibilité de revoir les prix d'achat tous les quatre mois en fonction de la conjoncture. La contractualisation des approvisionnements en bacon est également à l'étude (Les Marchés, 2014) ${ }^{23}$.

22. Agra Alimentation (2013). La contractualisation selon McDonald's. Agra Alimentation, $\mathrm{n}^{\circ} 2255$, septembre, pp. 1-5.

23. Les Marchés (2014). McDonald's se préoccupe des coûts de production du jambon. Les Marchés Hebdo, $\mathrm{n}^{\circ} 233,6$ juin, p. 8. 
Des sociétés de restauration collective, qui proposent des prestations dans un large cadre (collectivités, enseignement, santé, etc.), mettent en place avec leurs fournisseurs industriels des contrats d'approvisionnement fixant les prix et les quantités qui peuvent porter sur de longues périodes (plusieurs mois, un an, voire plusieurs années). Les menus des prestations proposés sont alors établis très longtemps à l'avance. Certaines caractéristiques du contrat sont orientées par le client final de la prestation (provenance de la viande, etc.).

Par ailleurs, au stade de la distribution, le développement de produits sous Marque de distributeur (MDD) fait aussi l'objet d'un contrat entre une enseigne et un industriel sur l'élaboration des produits, avec des modalités particulières de fixation des prix. L'industriel est considéré comme « prestataire de services ». Ces marques occupent une place importante dans la filière porcine. Hors hard discount, les MDD ont représenté environ $50 \%$ des tonnages de charcuterie en libre-service achetés par les ménages en 2013.

Les initiatives récentes mises en place à l'aval de la filière porcine permettent d'améliorer l'adéquation de l'offre des industriels à la demande des transformateurs, distributeurs ou consommateurs finaux sur des marchés spécifiques. Dans la recherche de satisfaction de demandes plus larges de consommateurs par des produits adaptés, la contractualisation permettrait aux entreprises de mettre en place des stratégies de développement de leur offre sur un plus long terme. Des expérimentations, menées par un nombre restreint d'entreprises sur des couples produits-marchés bien définis, pourraient être une voie pour en évaluer l'intérêt.

\section{$*$}

$* *$

Au sein d'un marché du porc qui se raisonne à l'échelle européenne, la commercialisation des porcs en France est très organisée. Les porcs sont vendus en grande majorité par des Organisations de producteurs (OP) selon des modalités transparentes. Deux essais de Contrats à livraison différée (CLD) à prix fixe des porcs, dont l'objectif était de garantir une marge, ont eu peu d'écho jusqu'à présent. À l'heure actuelle, les contrats proposés en France ne permettent pas de contrebalancer la variabilité des prix, car ils sont d'une durée trop courte pour être effectifs pour les éleveurs. Du point de vue des abatteurs, les volumes d'approvisionnement sont encore satisfaisants et les modes de commercialisation à l'aval sont peu favorables à une fixation des prix à moyen terme.

À l'étranger, les CLD sont utilisés plus fréquemment, pour garantir un débouché (États-Unis, Angleterre, Italie), garantir un prix correspondant à la réalité du marché au moment de la vente (États-Unis, market formula) ou encore limiter les fluctuations de prix (États-Unis, other market formula).

Pour la France, il reste à déterminer, par une évaluation fine, les meilleurs remèdes aux effets de la variabilité des prix du porc sur les différents acteurs, contractualisation ou autres modes de sécurisation des revenus ou des marges.

Mais il est certain qu'une coordination accrue entre les différents maillons permettrait de mieux adapter les produits offerts aux besoins, concourant certainement à une meilleure compétitivité du secteur. 


\section{Encadré. L'observatoire de la formation des prix et des marges des produits alimentaires et la production d'informations sur la transmission des prix et des coûts dans les filières animales : exemple en filière porcine Philippe BOYER • Fabien DJAOUT • Amandine HOURT}

\section{Méthodes des travaux de l'observatoire de la formation des prix des produits alimentaires}

L'Observatoire de la formation des prix et des marges des produits alimentaires (OFPM) ${ }^{24}$ met en place des approches comparables dans différentes filières animales (produits ajoutée par les secteurs de l'industrie agroalimentaires et marges commerciales où $r_{i}$ désigne le rapport entre la quantité vendue par un stade donné $i$ et la quantité de matière première agroalimentaire achetée et $P_{i}$ désigne le prix unitaire du produit vendu par le stade $i$ ( $P_{1}$ étant alors le prix du produit agricole).

$$
P_{n}=\left[P_{n}-\frac{P_{n-1}}{r_{n}}\right]+\left[\frac{P_{n-1}}{r_{n}}-\frac{P_{n-2}}{r_{n} r_{n-1}}\right]+\ldots\left[\frac{P_{i}}{r_{n} \ldots r_{i+1}}-\frac{P_{i-1}}{r_{n} \ldots r_{i}}\right]+\ldots+\frac{P_{1}}{r_{n} \ldots r_{2}}
$$

laitiers, viande bovine, porcine, volailles) et végétales (fruits et légumes, blé tendre/pain, blé dur/pâtes alimentaires). Ces approches permettent de décomposer les prix au détail en fonction des marges aux différents stades de la filière, de mesurer les coûts et de fournir des indicateurs de transmission des coûts. Elles reposent sur le schéma de travail technique unique suivant et s'appliquent de manière générale dans toutes les filières agroalimentaires suivies par l'observatoire.

Tout d'abord, un produit ou plusieurs produits de grande consommation sont retenus dans chaque filière, et leur prix au détail $P_{n}$ est décomposé en fonction de la valeur de la matière première d'origine agricole et des « marges brutes » de chaque stade en aval de la production agricole, éléments de valeur

24. L'OFPM est une commission administrative consultative créée par la loi n²010-874 du 27 juillet 2010 de modernisation de l'agriculture et de la pêche aux fins «d'éclairer les acteurs économiques et les pouvoirs publics sur la formation des prix et des marges au cours des transactions au sein de la chaîne de commercialisation. »Y participent les représentants des organisations professionnelles de chaque maillon des filières, les organisations de consommateurs, les services de la statistique publique, les administrations de l'agriculture et de la consommation. Il est présidé par Philippe Chalmin (professeur, Université Paris-Dauphine) et s'appuie sur l'établissement public FranceAgriMer.
Ensuite, le niveau et les évolutions des marges brutes en aval de la production agricole sont expliqués par des éléments de comptabilité générale et analytique. Pour l'analyse des marges brutes de la grande distribution, l'observatoire conduit, avec la participation des principales enseignes d'hyper- et supermarchés, une enquête spécifique sur leurs comptes analytiques par rayon alimentaire ; les charges prises en compte étant les intrants assurant le fonctionnement des magasins leur approvisionnement par les centrales d'achat ${ }^{25}$. Par ailleurs, la formation de la valeur dans le maillon agricole est appréhendée au travers de l'analyse des coûts de production.

Dans cet article, on applique la méthodologie à la filière porcine française pour analyser la formation et la transmission des prix dans la filière porc frais et charcuterie de porc.

\section{Prix, coûts et marges dans la filière des produits frais du porc}

Dans la filière de la viande de porc « frais », la matière première agricole représente en

25. Par exemple, les loyers prélevés sur le CA des magasins et perçus, selon les enseignes, par des sociétés de portage du foncier commercial du groupe propriétaire de l'enseigne ou par des entités de type SCI investies par des exploitants de magasins, sont comptés comme des charges de ce périmètre, qui ne s'identifie donc pas à une consolidation financière. 
moyenne sur 2000-2013, $41 \%$ du prix (hors TVA) des produits de longe au détail en GMS (graphique 1), avec des variations qui suivent, mais de façon amortie, celles du prix du porc entrée abattoir. L'abattage-découpe contribue pour moins de $9 \%$ en moyenne sur la période 2010-2013 à la formation du prix au détail hors TVA des produits de la longe (côtes, rôtis, escalopes de porc) et cette part est en baisse de 2010 à 2013, ce maillon n'ayant pas transmis intégralement la hausse du prix de sa matière première dans le prix de ses livraisons à la grande distribution.

Graphique 1. Décomposition du prix moyen au détail en GMS des produits de longe de porc en UVCM (2010-2013)

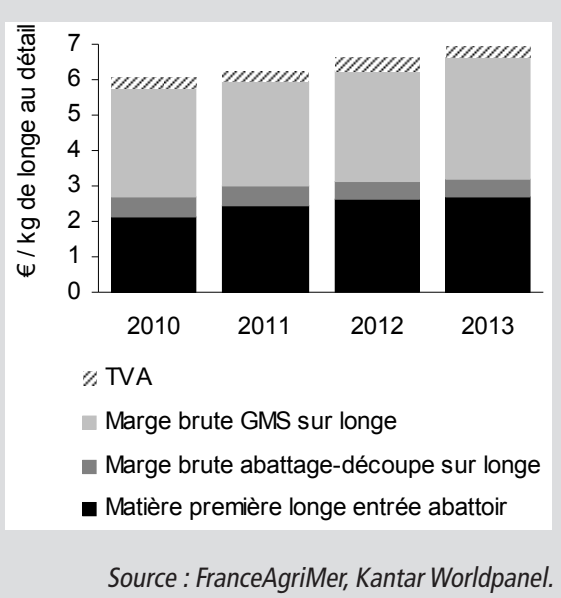

Graphique 3. Coût de l'abattage et de la découpe du porc (2010-2012)

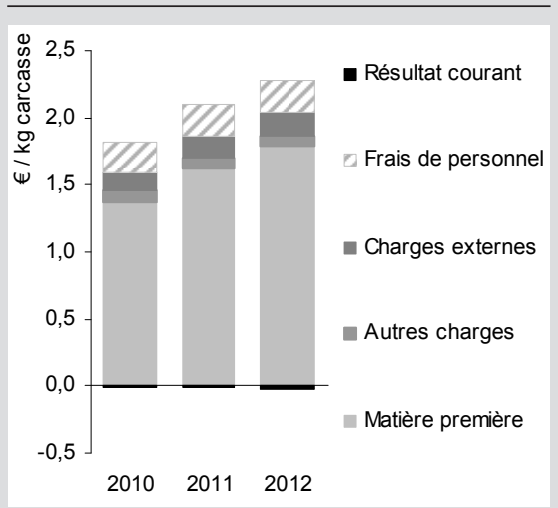

Source : FranceAgriMer d'après panel d'entreprises.
La marge brute de cette dernière représente en moyenne $51 \%$ du prix au détail hors TVA sur la période $2010-2013$ et cette part paraît en légère hausse de $2010(50,1 \%)$ à 2013 $(51,4 \%)$.

Les comptes des différents maillons montrent, s'agissant de la production agricole (graphique 2), le poids croissant du coût de l'alimentation des porcs. Dans l'abattage-découpe de porcs (graphique 3), la hausse des coûts sous l'effet de l'augmentation des charges de matière première, non transmise intégralement dans les prix de

Graphique 2. Coût de production du porc (2010-2012)

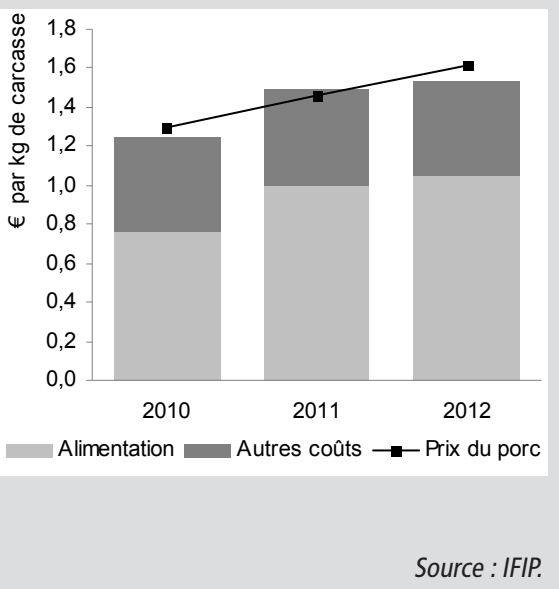

Graphique 4. Comptes moyens des rayons boucherie et charcuterie en GMS (2012)

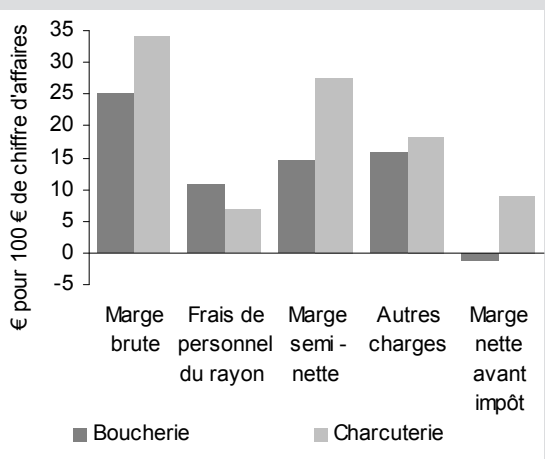

Source : OFPM - FranceAgriMer d'après enseignes. 
vente, entraîne un résultat négatif de 2010 à 2013 dans le panel d'entreprises suivies par FranceAgriMer.

Le rayon boucherie (dans lequel est vendue, entre autres viandes fraîches, la viande de porc) présente un taux de marge brute de $25 \%$. Les charges de main-d'œuvre spécifique du rayon représentent près de $11 \%$ du chiffre d'affaires, la grande distribution assurant une part significative des dernières phases du " démontage » des carcasses en morceaux à consommer. En comptabilisant les autres charges des GMS (personnel de caisse, foncier, frais financiers...) au prorata des rayons, le rayon boucherie présente une contribution négative au résultat net de l'ensemble de l'activité de vente au détail des enseignes (graphique 4).

\section{Prix, coûts et marges dans la filière des produits de charcuterie de porc}

La grande diversité des produits de charcuterie a contraint l'OFPM à ne suivre qu'un seul type d'articles vendus en GMS : l'ensemble des jambons cuits. La décomposition du prix au détail (graphique 5) met en évidence des résultats similaires à ceux observés pour le porc frais aux stades de la production agricole et de la première transformation (abattage-découpe). La marge brute de la

Graphique 5. Décomposition du prix moyen au détail en GMS du jambon cuit libre-service et à la coupe (2010-2013)

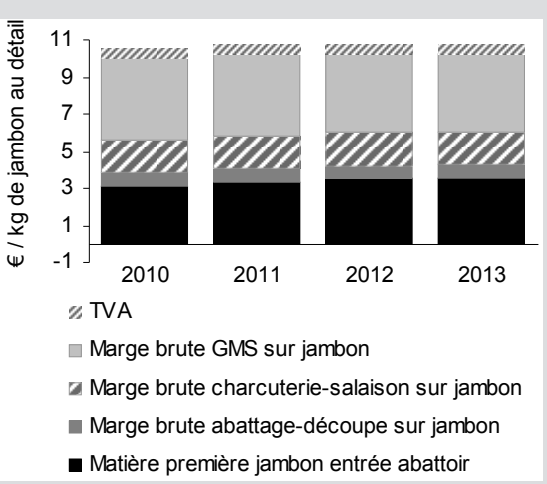

Source : FranceAgriMer, Kantar Worldpanel. $2^{\mathrm{e}}$ transformation (industrie de charcuteriesalaisonnerie) représente en moyenne sur 2000-2013 $16 \%$ du prix du détail hors TVA du produit. Cette marge est plutôt stable sur la période 2010-2013 alors que cette période est caractérisée par la hausse des prix de la matière première ; la situation des autres produits de l'industrie de la charcuterie est moins favorable et les comptes (graphique 6) montrent une baisse du taux de profit avant impôt (résultat courant) sur le chiffre d'affaires du secteur.

Avec près de $6 \%$ de marge nette sur le chiffre d'affaires, le rayon charcuterie est l'un des plus profitables des rayons frais des enseignes (graphique 4).

\section{Transmission des coûts dans la filière porc}

La transmission des prix et des coûts dans les chaînes de transformation et de commercialisation est analysée de façon synthétique par l'observatoire à l'aide des indicateurs suivants :

- l'évolution de 2005 à mi-2013 (en indice base 2005) du prix de la matière première du stade considéré (selon le stade : aliment des porcs, carcasse entrée abattoir, pièce sortie découpe, article vendu à la grande distribution par l'industriel) ;

Graphique 6. Structure des charges de l'industrie de charcuterie-salaison (2010-2012)

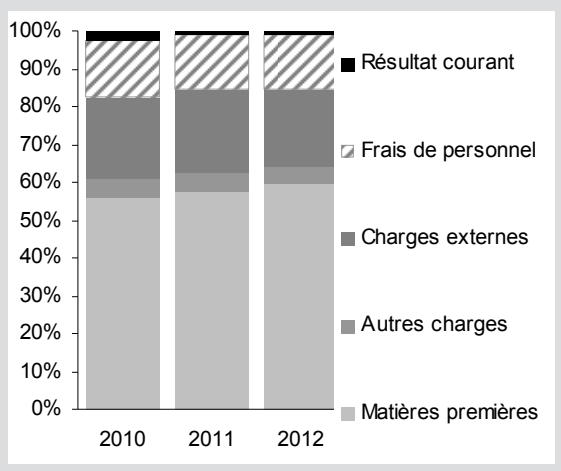

Source : Banque de France. 
- l'évolution du prix observé du produit élaboré par ce stade ;

- l'évolution du prix de ce même produit, simulé en maintenant la marge brute sur matière première à un niveau de référence en euros constants, soit la moyenne de cette marge sur 2001-2004, majorée de l'effet de la hausse des prix des autres charges à couvrir par cette marge brute.

Graphique 7. Prix du porc entrée abattoir, observé et simulé à marge brute de l'élevage maintenue au niveau 2001-2004

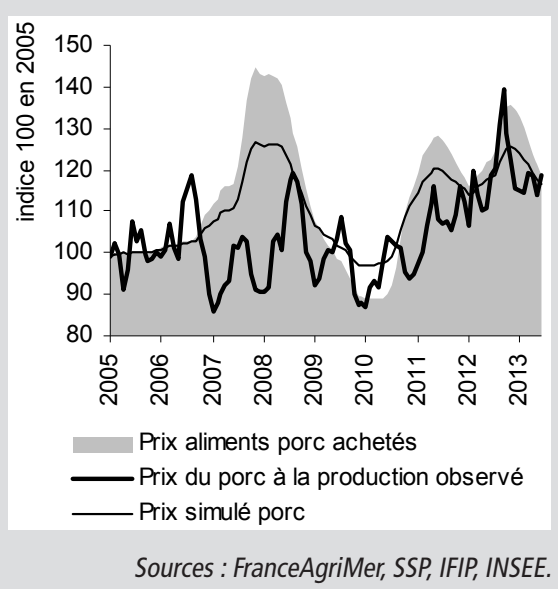

Graphique 9. Prix du jambon sortie $2^{\circ}$ transformation, observé et simulé à marge brute de la $2^{\circ}$ transformation maintenue au niveau 2001-2004

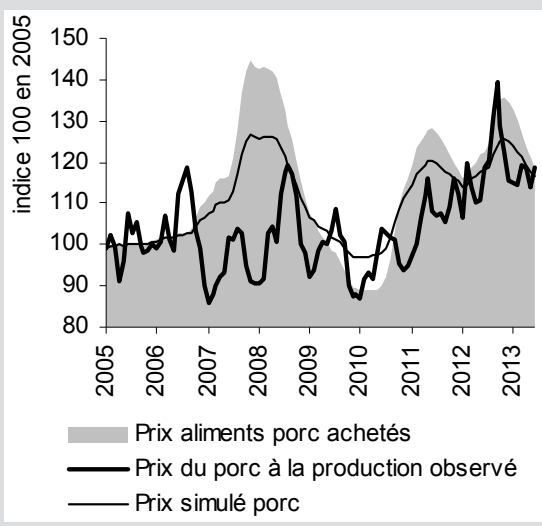

Sources : FranceAgriMer, INSEE.
Le coût de l'alimentation représente près de $70 \%$ du coût de production moyen en élevage selon l'IFIP en 2012. Son évolution est largement déconnectée de celle du prix du porc (graphique 7), du moins avant 2010. Ensuite, le prix des matières premières de l'alimentation animale et le prix du porc sont tirés parallèlement à la hausse par la demande mondiale, et notamment asiatique.

Graphique 8. Prix de la pièce de jambon sortie découpe, observé et simulé à marge brute de la découpe maintenue au niveau 2001-2004

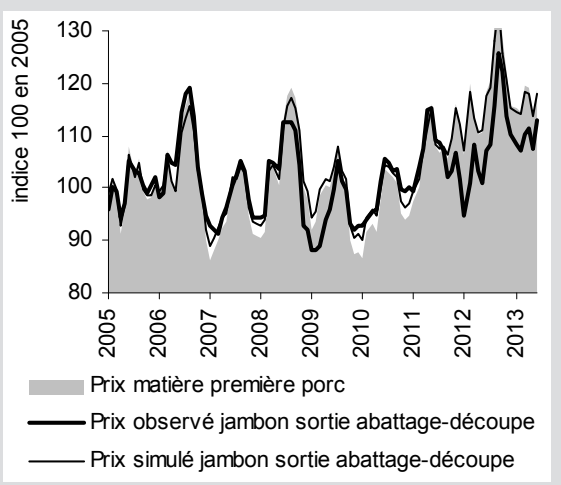

Sources : FranceAgriMer, INSEE.

Graphique 10. Prix du jambon cuit au détail, observé et simulé à marge brute de la distribution en GMS maintenue au niveau 2001-2004

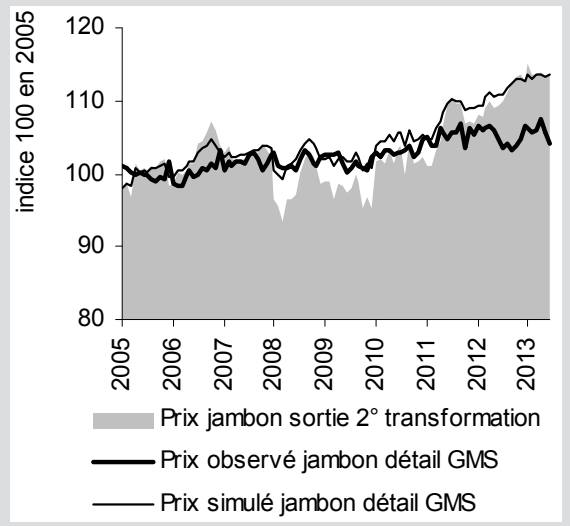

Sources : FranceAgriMer, INSEE. 


\section{FAITS ET CHIFFRES}

Dans les maillons en aval de la production, prix observés et prix simulés suivent des évolutions très proches. Ces évolutions sont presque identiques dans l'abattage-découpe. Dans ce secteur, les prix du porc entrée abattoir tendent à être intégralement transmis, sauf à certaines périodes (début 2012 et 2013) où le prix effectif décroche sensiblement (graphique 7).
L'industrie de la charcuterie-salaisonnerie répercute ses coûts dans le prix du jambon sortie usine de façon lissée (graphique 8). En GMS, depuis 2011, le prix moyen au détail des jambons cuits parait amortir la hausse des prix amont (graphique 10). 